\title{
Engagement in Online Medical Communities of Practice in Healthcare: Analysis of Messages and Social Networks
}

\author{
David Fowler ${ }^{1}$, Martin Szomszor ${ }^{1}$, Simon Hammond ${ }^{1}$, John Lawrenson ${ }^{2}$, and Patty \\ Kostkova ${ }^{1}$. \\ ${ }^{1}$ City eHealth Research Centre, City University, London, UK \\ ${ }^{2}$ Optometry and Visual Science, City University, London, UK \\ David.Fowler.1@city.ac.uk
}

\begin{abstract}
We report on preliminary analysis on user engagement in two online medical communities of practice. Despite the communities being independent of each other, and dealing with different domains (field epidemiology and therapeutic prescribing for optometrists), there are some clear similarities in the networks of users, and in patterns of replies to user postings. We also draw some initial conclusions to help maintain user engagement in these and similar sites, and we suggest some future lines of research.
\end{abstract}

\section{Introduction}

Professional communities of practice (CoPs) have been the cornerstone for sharing scientific knowledge and professional discourse. The internet has dramatically changed the way communication and peer networking is managed: little overhead and flat structures, easy online recording of scientific discussions, higher frequency of postings, and virtually unlimited geographical coverage of the CoPs. However, online communities may be vulnerable to stagnation and failure if the support tools are not suitable, or if key members of the community are not able to take an active role.

\subsection{Communities of practice}

The term "community of practice" has many definitions, although it originates in the work of Lave and Wenger [1]. We will use the definition of CoPs as "groups of people who share a concern, a set of problems, or a passion about a topic, and who deepen their knowledge and expertise in this area by interacting on an ongoing basis." [2] (also cited in [3]). CoPs may be deliberately created or spontaneously emerge, and be highly structured or informal [3].

\subsection{The two communities: FEM Wiki and MSU}

We examine two independent CoPs developed around medical scientific internet portals: FEM Wiki (http://www.femwiki.com), dealing with field epidemiology, and 
Medicines Support Unit for Optometrists (MSU, http://www.med-support.org.uk), supporting therapeutic prescribing by optometrists. The user bases are geographically dispersed (mainly throughout the UK for MSU and throughout Europe for FEM Wiki). Both sites provide centrally authored information to specialists, and have means for user discussion. Each was created to order, but FEM Wiki is more highly structured than MSU. In FEM Wiki, users can directly edit the content, but to guarantee quality, changes must be approved before the changes are made official. In MSU, changes can be suggested informally via the forum.

\section{Social Network Analysis}

We collected the messages that were posted on the discussion forums of the communities, and extracted networks of users. Each node corresponds to a user, with arcs linking the nodes of users who were involved in the same discussion (Fig. 1).
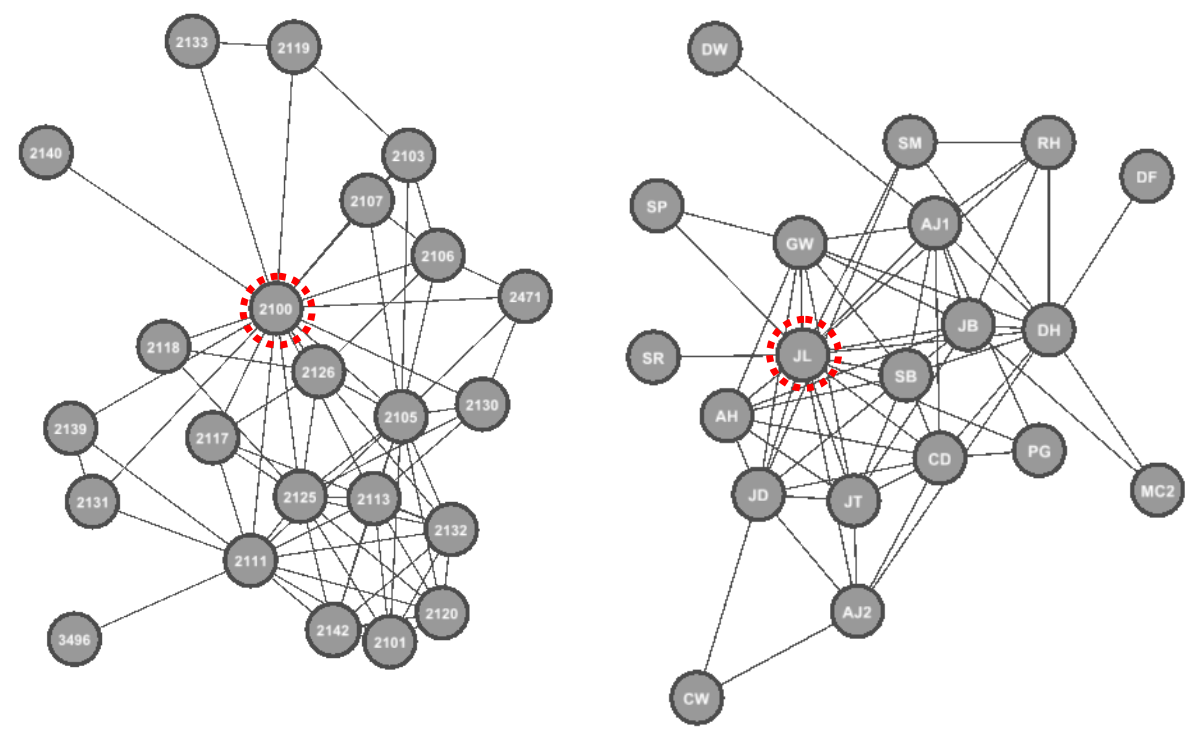

Fig. 1. The user networks extracted from FEM Wiki (left), and MSU (right). The nodes with most connections are highlighted.

Fig. 2 shows the number of connections for each node in the networks, and Table 1 summarises some key statistics. Each network has a number of users who are involved in many discussions; these seem to be mainly senior project leaders or administrators. There is an almost linear decline to users who were only involved in one or two discussions (possibly they only had a specific question that was answered to their satisfaction). Although the networks that are extracted are not a complete picture of the knowledge sharing activities in the communities (for example, members may share knowledge in person or via other media and the network does not measure 
the quality of contributions), it may give a reasonable approximation. Users with many connections are involved in many discussions, and therefore may have more knowledge and experience to share.

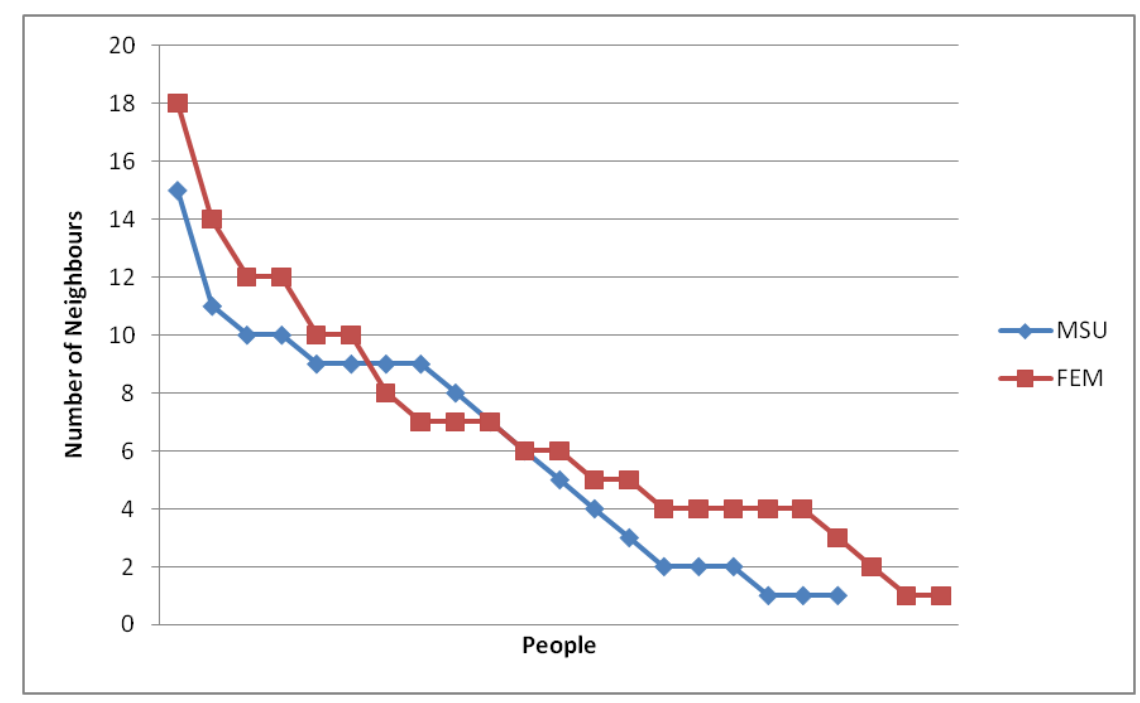

Fig. 2. The numbers of neighbours for each node in Fig. 1.

Table 1. Summary statistics for the two communities.

\begin{tabular}{lcc}
\hline & FEMWiki & MSU \\
\hline Nodes & 23 & 20 \\
Edges & 73 & 62 \\
Average Degree & 6.348 & 6.200 \\
Diameter & 3 & 4 \\
Average Path Length & 1.798 & 1.816 \\
Graph Density & 0.289 & 0.326 \\
\hline
\end{tabular}

\section{Message analysis}

In addition to examining the connections between users, we also looked at the characteristics of the messages. There were striking similarities between FEM Wiki and MSU in the distribution of replies to messages. Table 2 shows that the majority of posts have a small number of replies (the median is 2 for both communities). This seems to be typical behaviour for online forums, e.g. [4]. 
Table 2. Summary statistics for the numbers of replies to FEM Wiki and MSU posts.

\begin{tabular}{ccc}
\hline & FEM Wiki (33 posts) & MSU (45 posts) \\
\hline Min & 0 & 0 \\
Q1 & 1 & 1 \\
Median & 2 & 2 \\
Q3 & 3 & 3 \\
Max & 18 & 16 \\
\hline
\end{tabular}

\section{Discussion and Future Work}

We have shown that there are underlying similarities in the user network structure and distribution of numbers of replies to posts of two independent online CoPs. The two sites also vary in their organisational structures and editing processes, so these results might suggest some properties that are shared more widely between online CoPs. This should provide some useful lines of enquiry, although it will require access to data from a larger number of online CoPs. We will also need to investigate how the properties of CoPs vary with size, as our examples were both in the small to medium range.

The type of analysis in this paper may be helpful in identifying users whose contributions are critical to keeping an online community active. If such users become less active (for example, through pressures of other work), there is a risk that the community will stagnate, and lose other users. There is some evidence that this has happened recently with the MSU site (although with MSU there was another possible cause for loss of activity: a spam attack on the discussion forum may have driven away some users).

We are interested in tracking the activity of online CoPs over time to see how the user networks vary, investigating what factors may affect the activity, and whether there is an identifiable "critical point" at which community activity breaks down. We are currently redesigning the MSU site, and plan to promote the site again to existing and prospective users in order to increase activity.

Finally, we plan to investigate the factors that affect the user response to forum messages. Section 3 showed high level similarities, and it will be interesting to see which types of posts attract most discussion, and to draw comparisons between sites.

\section{References}

1. Lave, J., Wenger, E.: Situated Learning: Legitimate Peripheral Participation (Cambridge University Press, Cambridge, 1991).

2. Wenger, E., McDermott, R., Snyder, W.M.: Cultivating Communities of Practice: A Guide to Managing Knowledge (Harvard Business School Press, Boston, MA, 2002).

3. Hara, N., Shachaf, P., Stoerger, S.: Online communities of practice typology revisited, Journal of Information Science vol. 35 no. 6, 740--757 (2009)

4. Mishne, G., Glance, N.: Leave a reply: An analysis of weblog comments, In: Third Annual Workshop on the Weblogging Ecosystem (part of WWW2006) (2006) 